Historic, Archive Document

Do not assume content reflects current scientific knowledge, policies, or practices. 


\section{THE HOME OF}

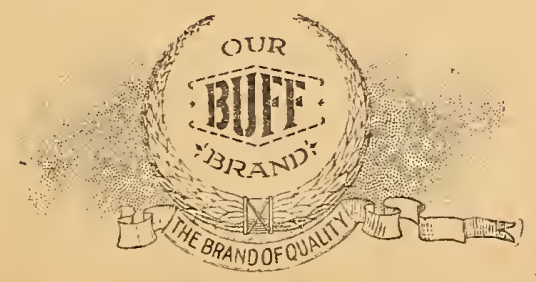

\section{SEEDS}

\section{JOHN J. BUFFINGTON \& CO. SEED MERCHANTS}

BALTIMORE

-

MARYLAND

\section{BUFF BRAND SEEDS}





\section{JOHN J. BUFFINGTON \& CO. \\ WHOLESALE SEED MERCHANTS \\ $108-118$ LIGHT STREET}

BALTIMORE, MD., JANUARY 28, 1927.

There has been no material change in market conditions during the week.

Both inquiries and orders show improvement. We expect an active demand after early part of February provided the weather is favorable. We can supply you with both the DOMESTIC and FRENCH grown Red Clover to advantage. The FRENCH SEED we consider splendidly adapted to your conditions. Will appreciate your inquiries and send samples when desired-PHONE, WIRE or WRITE US, WE CAN PLEASE YOU-QUALITY, PRICE, SERVICE.

We do not handle Italian or South Euronean grown Red Clover or Alfalfa.

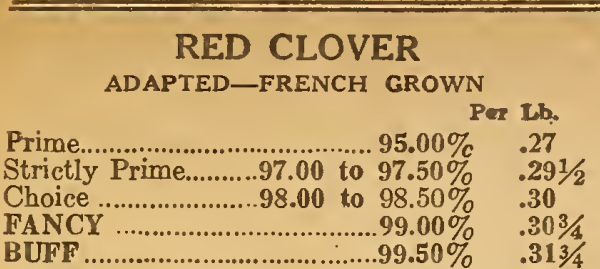

IN SACKS OF ABOUT 220 LBS. EACH FANGY................. 98.50 to $99.00 \% \quad .30$

RED CLOVER UNITED STATES GROWN

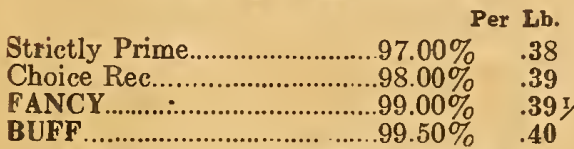

\section{SAPLING OR MAMMOTH} UNITED STATES GROWN Per Lb. Choice...................................98.00\% $\quad \mathbf{. 4 0}$

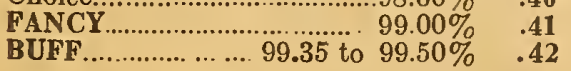
ALSIKE CLOVER Choice. ................95.00 to $96.00 \% \quad$ Per Lb. UTFF. 98.00 to $98.50 \%$

ALFALFA

UNITED STATES GROWN Per Lb. Choice ................................... 98.50\% $99.00 \%$ BUFF ................................. $99.00 \%$ $99.50 \%$

KANSAS ALFALFA Per Lb. ................ 99.50\% $\quad .20$ NORTHWESTERN ALFALFA BUFF ..............99.50\% $\%^{\text {Par Lo }} .201 / 2$ CANADIAN VARIEGATED BUFF..................... Per Lb. GRIMM'S ALFALFA CLOVER BUFF............Certified........... 99.50\% $\quad .34$ CRIMSON CLOVER

FANCY Per Lb. WHITE CLOVER

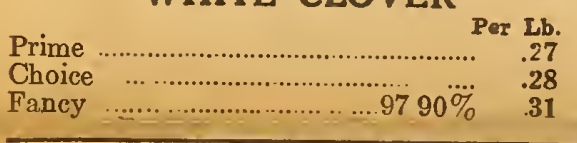

\section{HuMogerir}

Insures Duicker Catch-Bigger Crops

\section{CLUVERS}

$\begin{array}{lcr} & \text { Wholessle } & \text { Retail } \\ 21 / 2 \text { Bu. Onit } & \$ 1.50 & \$ 2.25 \\ 1 \text { Bu. Onit } & .67 & 1.00\end{array}$

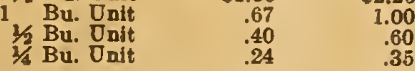

SOY BEANS - COW PEAS - VETCHES

$\begin{array}{ccc} & \text { Wholesale } & \text { Retail } \\ 5 \text { Bu. Unit } & \$ 1.50 & \$ 2.25 \\ 1 \text { Bu. Unit } & : 40 & .60 \\ \text { 1/2 Bu. Unit } & .24 & .35\end{array}$

State desired variets.

Posters and Lenflets on request

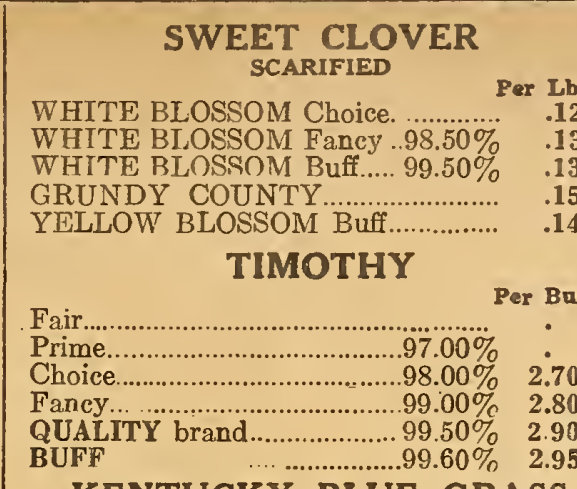

\section{KENTUCKY BLUE GRASS}

Choice Kentucky................80.00\% ${ }^{\text {Per }}{ }^{\mathbf{L b}}$

Fancy Kentucky ..................85.00\% $\quad .24$

Canadian

\section{ORCHARD GRASS}

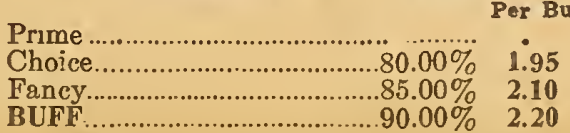

\section{RED TOP/HERD GRASS} BUFF Fancy............ $92.00 \%$

Choice Unhulled ....................................

Faney Unhulled …................................... $\quad .16$

RYE $\cdot$ GRASS

Perennial................................98.50\% $\begin{array}{lll}\text { Italian } & \ldots . \ldots \ldots \ldots \ldots \ldots \ldots . . . . . . . .98 .50 \% & .09 \\ \text { Paceys Short Seeded .............98.50\% } & .141\end{array}$

CRESTED DOGSTAII

HARD FESCUE

SHEEP FESCUE.

RED FESCUE

\section{PERMANENT PASTURE}

For Upland.

Per Lb.
.12 to .14

For Lowland

GERMAN MILLET

2 to .14

Choice

Fancy

Per Bu.

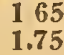

TENNESSEE Fancy............................. 1.80

WHITE WONDER (large growth).
HUNGARIAN

SORGHUM OR CANE

Fancy Amber

Fancy Orange..................................................
Fancy....................................85.00\% 2.10

Choice Fancy........................90.00\% Per Lb.

VARIOUS SEEDS Par Lb

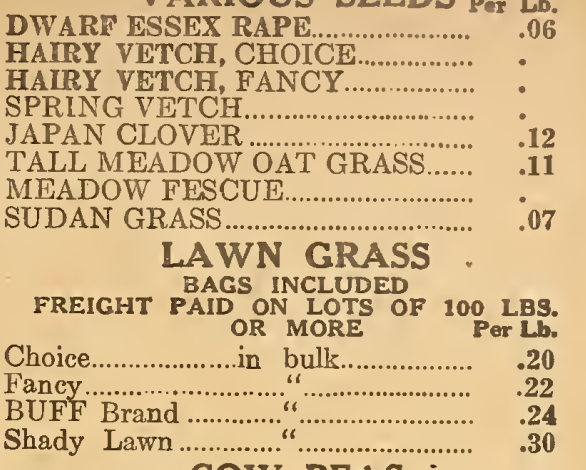

\section{COW PEAS}

Mixed.

RECLEANED, SACKS FREE Per Bu.

Whippoorwill Mixed.

Brown Whippoorwill...........

Gray Whippoorwill

Blacks.......

New Era

Brabham

Clay.

Gray Crowder.

COW PEAS and SOJA BEANS Mixed.

\section{SOJA BEANS}

RECLEANED, SACKS FREE Per B

Mammoth Yellow.

Manchu

Waredo.

Wilson.

Hollybrook

Tar Heel.

CANADA FIELD PEAS

Choice

AMERICAN GROWN PET BE.

Fancy

\section{SEED GRAIN}

Maryland Winter Seed Rye.

Rosen Seed Rye

Abruzzi Seed Rye

Buckwheat, Japanese

FULTZ WHEAT

Leafs Prolific Wheat

China Wheat

BARLEY.

Winter Seed Oats (Virginia Gray)

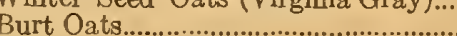

ENSILAGE CORN

\section{(Southern White}

\section{MISCELLANEOUS FEEDS}

ALASKA PEAS (Sealed).... 6.60 SEED POTATOES (MAINE GROWN)

Irish Cobblers................11 Peck Sack 7.00 Early Rose......................11 Peck Sack Red Bliss.....................11 Peck Sack 7.00 Crown Jewell.

Green Mountain.............11 Peck Sack

\section{BUFF BRAND POULTRY FEEDS} SCRATCHING SIZE

10 sa.ck lots......................per $100 \mathrm{lbs} . \quad 2.35$ Per 100 lb. sa.ck.................per 100 lbs. 2.40 CHICK SIZE

10 sa.ck lots......................per 100 lbs. 250 Per 100 lb. sack........... per 100 lbs. 255
DRY MASH.. 100 lbs. 2.50

Beef Scraps..............50\% per $100 \mathrm{lbs} . \quad 375$

Crushed Bone....................per 100 lbs. 3.00

Meat Meal $50 \%$ per 100 lbs 3.75

Deat Mes.....50\% per 100 lbs. $\mathbf{3 . 7 5}$

Digester Tankage..60\% per 100 lbs. 3.75

Sunflower......................................per lb. .05

Buckwheat.

Vetch

.

(bs. 2.30

Canada Field Peas.....................per lb.

Alfalfa Meal............................ 100 lbs. 2.20

CRUSHED OYSTER SHELL

Ton lots....

10 sack lots......... per lb. .03

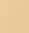

4




\section{LAWN GRASS AND PASTURE MIXTURES}

\section{QUALITY IS OF GREATEST IMPORTANCE}

OUR LAWN AND PASTURE MIXTURES ARE COMPOSED OF SEEDS OF THE BEST QUALITY AND IN PROPER PROPORTIONS.

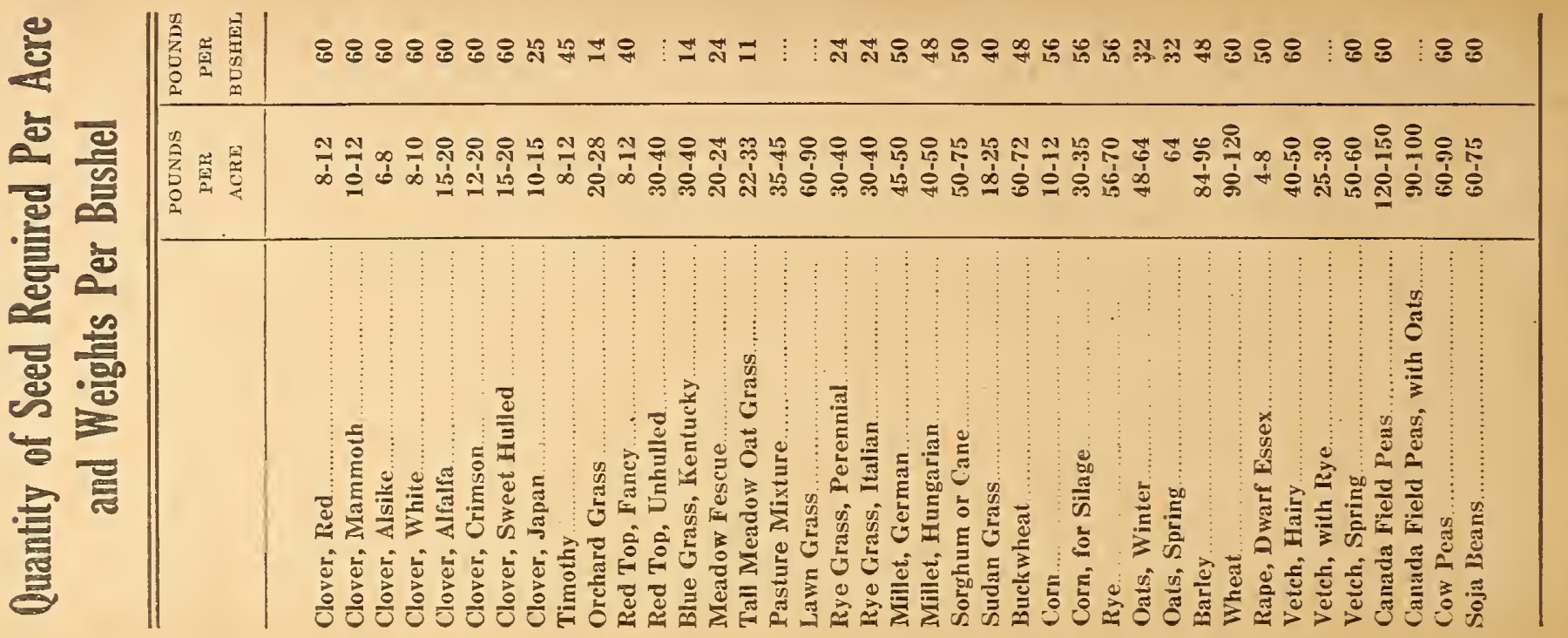

